\title{
Bioequivalence and Dose Proportionality of Inhaled Fluticasone Furoate
}

\author{
Ann Allen ${ }^{1 *}$, Jo Bal ${ }^{2}$, Alison Moore ${ }^{2}$, Sally Stone ${ }^{2}$ and Lee Tombs ${ }^{3}$ \\ ${ }^{1}$ Clinical Pharmacology Modelling and Simulation, GlaxoSmithKline R\&D, Stevenage, United Kingdom \\ ${ }^{2}$ GlaxoSmithKline R\&D, Stockley Park, United Kingdom
}

${ }^{3}$ Synergy, Slough, Berkshire, United Kingdom

\begin{abstract}
Fluticasone furoate (FF), a new inhaled corticosteroid delivered via the ELLIPTA dry powder inhaler, is being developed as a once-daily inhaled treatment for asthma. Study 1 was a part-randomised, open-label, four-way crossover, single- and repeat-dose study in healthy subjects $(n=36)$ to assess whether the systemic exposure of FF increased proportionately across different strengths of FF $(50 \mu \mathrm{g}, 100 \mu \mathrm{g}$ and $200 \mu \mathrm{g})$ and to determine the absolute bioavailability of FF inhalation powder. Study 2 was a randomised, open-label, replicate, six-way crossover, single-dose study in healthy subjects $(n=30)$ to determine the bioequivalence of $F F$ inhalation powder (single-strip configuration) compared with FF inhalation powder (two-strip configuration) and with fluticasone furoate/vilanterol (FF/VI) inhalation powder. A population pharmacokinetic analysis was also conducted on sparse samples collected from asthma patients in five Phase III studies conducted with FF/ VI or FF.

Overall, FF systemic exposure, as measured by single-dose $\mathrm{AUC}_{(0-\infty)}$, was dose proportional whilst $\mathrm{C}_{\max }$ showed a less than proportional increase. Inhaled absolute bioavailability of FF was $14 \%$. Bioequivalence was not demonstrated for FF single-strip compared with either FF two-strip or FF/VI since the estimated $90 \%$ confidence interval for the ratio of adjusted geometric means for AUC and $\mathrm{C}_{\max }$ did not fall completely between 0.8000-1.2500. However, this difference was in line with the higher respirable mass delivered by the batch of single-strip product used in this study.

Although the formal bioequivalence study showed higher exposure with FF single-strip ELLIPTA compared with FF two-strip or FF/VI, the results of the population PK analysis of data in asthmatics show that there is no notable difference in systemic exposure between the FF configurations (single-strip, two-strip or FF/VI). In healthy subjects all treatments were generally safe and no new safety issues were apparent at these supra-therapeutic inhaled doses of FF (up to $1200 \mu \mathrm{g})$ and high intravenous FF dose $(250 \mu \mathrm{g})$.
\end{abstract}

Keywords: Bioavailability; Bioequivalence; Fluticasone furoate; Healthy subjects; Inhaled; Pharmacokinetics; Proportionality; NCT01669070; NCT01485445

Abbreviations: AE: Adverse Event; $\mathrm{AUC}_{(0-\mathrm{r})}$ : Area Under the Curve from Zero (Pre-Dose) to the Time of Last Common Measurable Time Point Within Subject; BLQ: Below the Limit of Quantitation; CI: Confidence Interval; $\mathrm{C}_{\max }$ : Maximum Plasma Concentration; COPD: Chronic Obstructive Pulmonary Disease; CV: Coefficient of Variation; DPI: Dry Powder Inhaler; FF: Fluticasone Furoate; FP: Fluticasone Propionate; ICS: Inhaled Cortisosteroid; KEDTA: Tri-potassium Ethylene Diamine Tetra-acetic Acid; LABA: Long Acting BetaAgonist; MAT: Mean Absorption Time; NA: Not Applicable; t': Time of Last Common Measurable Time Point Within a Subject; $t$ : Time to Maximum Observed Concentration; VI: Vilanterol; VPC: Visual Predictive Check

\section{Introduction}

Fluticasone furoate (FF) is a new inhaled corticosteroid (ICS) in development for the treatment of asthma and in combination with vilanterol (VI), a new long-acting beta ${ }_{2}$ agonist (LABA), for asthma and chronic obstructive pulmonary disease (COPD). FF is structurally distinct from fluticasone propionate (FP) $[1,2]$ and has demonstrated a longer duration of action than FP in vitro [3]. Once-daily dosing of FF is non-inferior to the same total dose given twice daily [4], and oncedaily doses of FF $100 \mu \mathrm{g}$ and $200 \mu \mathrm{g}$ are effective in improving lung function and asthma symptoms in patients with asthma uncontrolled on low- or mid-dose ICS, with an acceptable safety profile [5-8]. Furthermore, FF $200 \mu \mathrm{g}$ once-daily has been shown to be non-inferior to FP $500 \mu \mathrm{g}$ twice-daily on trough $\mathrm{FEV}_{1}$ over 24 weeks in patients with moderate-to-severe asthma [9].

The pharmacokinetic, pharmacodynamic and safety profiles of FF administered as the FF/VI combination have been described in healthy subjects as well as in patients with asthma and COPD [10-16].

The ELLIPTA ${ }^{\mathrm{TM}}$ (trade mark of the GlaxoSmithKline group of companies) dry powder inhaler (DPI) will be used to deliver both FF monotherapy and the FF/VI combination. The ELLIPTA DPI can be used for FF monotherapy (with either single strip [the developed formulation] or as a two-strip configuration [with matched VI placebo in the second strip]), or to deliver the FF/VI combination (as a twostrip configuration [i.e. with FF and VI in separate strips]).

Dose proportionality occurs when the clearance of a drug remains constant and exhibits linear time-independent pharmacokinetics over a range of doses, and can be demonstrated when the observed plasma concentrations increase proportionately to the dose

*Corresponding author: Ann Allen, Clinical Pharmacology Modelling and Simulation, GlaxoSmithKline, Stevenage, SG1 2NY, UK, Tel: +44 1438 766350; Fax: +44 1438 764591; E-mail: ann.allen@gsk.com

Received December 20, 2013; Accepted January 18, 2014; Published January 26, 2014

Citation: Allen A, Bal J, Moore A, Stone S, Tombs L (2014) Bioequivalence and Dose Proportionality of Inhaled Fluticasone Furoate. J Bioequiv Availab 6: 024032. doi: $10.4172 / \mathrm{jbb} .1000176$

Copyright: (c) 2014 Allen A, et al. This is an open-access article distributed under the terms of the Creative Commons Attribution License, which permits unrestricted use, distribution, and reproduction in any medium, provided the original author and source are credited. 
administered over the clinically relevant dose range. Understanding that the pharmacokinetics of the investigational product is linear over the concentration range of interest facilitates prediction of the effect of changing dose. Study 1 was performed to demonstrate dose proportionality of FF following single and repeat dose administration of three strengths of FF (50 $\mu \mathrm{g}, 100 \mu \mathrm{g}$ and $200 \mu \mathrm{g})$ via ELLIPTA. The absolute bioavailability of FF at the highest single dose administered in the study, $1200 \mu \mathrm{g}$ (given as six inhalations of FF $200 \mu \mathrm{g}$ ) was also determined. The bioavailability of inhaled FF predominantly represents absorption from the lung as the oral bioavailability of FF from the swallowed portion of the inhaled dose is negligible (approximately $1 \%$ ) [17].

The two-strip configuration has been used for the majority of FF monotherapy treatment arms in previous studies. Study 2 was conducted to determine the bioequivalence of FF monotherapy (single strip) compared with FF monotherapy (two-strip), and to determine the bioequivalence of FF monotherapy (single strip) compared with FF/ VI, administered via ELLIPTA. Due to the low systemic bioavailability of FF, and consequently the low blood levels after dosing at the clinical dose, it is not possible to assess bioequivalence, dose proportionality or absolute bioavailability at the proposed clinical doses (FF $100 \mu \mathrm{g}$, $200 \mu \mathrm{g}$ ). Consequently, in these studies multiple inhalations were administered in order to provide adequate pharmacokinetic data following single dose administration.

The FF pharmacokinetics in subjects with asthma was also evaluated using population pharmacokinetic methods on sparse samples collected from five Phase III studies conducted in subjects with asthma administered FF/VI or FF (single and two-strip) via ELLIPTA. Configuration (single or two-strip) was included as a potential covariate in the modelling procedure to investigate whether there were differences between the single and two-strip configurations.

\section{Methods}

\section{Study design and subjects}

Study 1: FF dose proportionality and absolute bioavailability (FFA115441; NCT01669070): This part-randomised, open-label, fourway crossover, single and repeat-dose study was conducted healthy male or female subjects $(n=36)$ to determine the dose proportionality and absolute bioavailability of fluticasone furoate administered as the single strip configuration ELLIPTA. Subjects were assigned to one of six treatment sequences $(\mathrm{ABCD}, \mathrm{ACBD}, \mathrm{BACD}, \mathrm{BCAD}, \mathrm{CABD}$ or $\mathrm{CBAD}$ ) where $\mathrm{A}=$ single dose of $300 \mu \mathrm{g} \mathrm{FF}$ ( 6 inhalations of $50 \mu \mathrm{g} \mathrm{FF}$ ) on Day 1, followed by $50 \mu \mathrm{g}$ FF once daily for 7 days: $\mathrm{B}=$ single dose of $600 \mu \mathrm{g}$ FF (6 inhalations of $100 \mu \mathrm{g}$ FF) on Day 1, followed by $100 \mu \mathrm{g}$ FF once daily for 7 days: $C=$ single dose of $1200 \mu \mathrm{g} F$ ( 6 inhalations of $200 \mu \mathrm{g} \mathrm{FF}$ ) on Day 1, followed by $200 \mu \mathrm{g}$ FF once daily for 7 days: $\mathrm{D}=$ single intravenous dose of FF $250 \mu \mathrm{g}(1 \mathrm{~mL}$ of $250 \mu \mathrm{g} / \mathrm{mL}$ in $100 \%$ propylene glycol administered at a constant rate of infusion over 20 minutes using a syringe and pump) that was administered in the final study period for all subjects. The inhaled and intravenous treatment periods had different dosing procedures, sampling requirements, pharmacokinetic sampling time points and treatment administration. Therefore, it was considered more practical to perform the study with all subjects receiving the intravenous treatment in groups to minimise any logistical issues. No significant statistical issues were expected in following this approach.

Study 2: FF Bioequivalence (FFA115440; NCT01485445):
This randomised, open-label, replicate, six-way crossover, singledose study in healthy male or female subjects $(n=30)$ was conducted to determine the bioequivalence of FF inhalation powder (single strip configuration) compared with FF inhalation powder (two-strip configuration) and compared with fluticasone furoate/vilanterol (FF/ VI) inhalation powder. Subjects were assigned to one of six treatment sequences (ABCBAC, BCACBA, CABACB, BACABC, CBABCA or $\mathrm{ACBCAB}$ ) where $\mathrm{A}=\mathrm{FF} 400 \mu \mathrm{g}(2$ inhalations of $200 \mu \mathrm{g})$, administered from ELLIPTA with no second strip (single-strip configuration): $\mathrm{B}=\mathrm{FF} 400 \mu \mathrm{g}(2$ inhalations of $200 \mu \mathrm{g})$ administered from ELLIPTA with a filled (lactose and magnesium stearate) second strip (twostrip configuration): C=FF/VI 400/50 $\mu \mathrm{g}$ ( 2 inhalations of 200/25 $\mu \mathrm{g}$ ) administered from ELLIPTA.

Both studies included non-smoking healthy male and female subjects aged 18 to 65 years with body mass index 18.5 to $29.0 \mathrm{~kg} /$ $\mathrm{m}^{2}$. Exclusion criteria included: treatment with an investigational drug within 30 days or five half-lives prior to the first dose of study treatment; use of prescription or non-prescription drugs and/or dietary supplements within 7 days (or 14 days if the drug is a potential enzyme inducer) or 5 half-lives (whichever is longer) prior to the first dose of study medication; history of alcohol/drug abuse or dependence within 6 months of the study; subjects who have suffered a lower respiratory tract infection within 4 weeks of the screening visit; electrocardiogram demonstrating corrected QT interval greater than $450 \mathrm{msec}$ at screening. Females who were pregnant or nursing were also excluded.

There was a washout period of at least 7 days but no more than 14 days between treatment periods for both studies. The duration for each subject was approximately 14 weeks and 11 weeks for Study 1 and 2, respectively.

Both healthy subject trials were conducted in compliance with Good Clinical Practice with the ethical principles that have their origins in the Declaration of Helsinki. The investigators obtained institutional review board approvals for the study protocols (Study 1 was conducted at PRA International, Hanzeplein 1 Entrance 23 Groningen Netherlands 9713 GZ, and Study 2 was conducted at Parexel International GmbH, Klinikum Westend, Haus 18, Spandauer Damm 13014050 Berlin, Germany). All subjects gave their written informed consent before participating in the trial.

\section{Asthma patient population pharmacokinetic analysis}

This Phase III, multicentre, randomised, double-blind, parallelgroup study was a 24 -week study in patients $(n=238$ randomised $)$ aged $\geq 12$ years with moderate-severe persistent asthma to evaluate the efficacy and safety of two strengths of once-daily FF: $100 \mu \mathrm{g}$ and $200 \mu \mathrm{g}$ (single-strip ELLIPTA) conducted at 40 centres in 6 countries (Argentina, United States, Chile, Russian Federation, Mexico, France [France did not participate in the PK aspects]).

\section{Pharmacokinetic evaluations}

Study 1: Venous blood samples (approximately $2 \mathrm{~mL}$ ) for analysis of FF plasma concentrations were collected in KEDTA tubes as follows: Inhaled dosing (Days 1 and 9): pre-dose and at 15, 30 and 45 minutes and $1,1.5,2,3,4,6,8,10,12,16,24$ hours and Day 9 only at 36 and 48 hours; Intravenous dosing: pre-dose and at 10, 20, 25, 30 and 45 minutes and 1, 1.5, 2, 3, 4, 6, 8, 10,12, 16, 24, 36 and 48 hours after the start of dosing.

Study 2: Venous blood samples $(3 \mathrm{~mL})$ for analysis of FF plasma 
concentrations were collected in KEDTA tubes pre-dose and at 15, 30 and 45 minutes, and 1, 1.5, 2, 3, 4, 6, 8, 10, 12, 24 and 36 hours after the start of dosing.

Asthma study: Single venous blood samples (4 mL) for analysis of FF plasma concentrations were collected in KEDTA tubes at Weeks 4 and 18 at pre-dose and between 45 and 75 minutes post-dose.

The blood samples for all studies were put on ice until centrifugation at $1500 \mathrm{~g}$ for approximately 10 minutes at $4^{\circ} \mathrm{C}$. Following centrifugation supernatant plasma was transferred into $1.4 \mathrm{~mL}$ matrix screw capped polypropylene tubes and frozen. All samples were stored at $-20^{\circ} \mathrm{C}$ or colder until shipment.

\section{Analytical methods}

Plasma samples $(150 \mu \mathrm{L}$ aliquot for Study 1 and $500 \mu \mathrm{L}$ aliquot for Study 2) from both Studies 1 and 2 were analysed for FF by solid phase extraction using $\left[{ }^{13} \mathrm{C}^{2} \mathrm{H}_{3}\right]-\mathrm{GW} 685698$ or $\left[{ }^{13} \mathrm{C}_{3}\right]-\mathrm{CC} 18781$ as internal standard for Study 1 and 2, respectively, followed by high performance liquid chromatography with tandem mass spectrometry using an Applied Biosystems API-3000 (Applied Biosystems/MDS Sciex, Foster City, USA). A gradient system using $5 \mathrm{mM}$ ammonium formate and methanol was run with column Poroshell $3.0 \times 50 \mathrm{~mm}, \mathrm{C} 182.7 \mu \mathrm{m}$, running at $50^{\circ} \mathrm{C}$. Plasma samples $(150 \mu \mathrm{L}$ aliquot) from the studies in subjects with asthma were analysed for FF by solid phase extraction using $\left[{ }^{13} \mathrm{C}^{2} \mathrm{H}_{3}\right]$-GW685698 as internal standard followed by high performance liquid chromatography with tandem mass spectrometry using an Applied Biosystems API-5000. A gradient system using $5 \mathrm{mM}$ ammonium formate and methanol was run with column ACE 50 *2.1 $\mathrm{mm}, \mathrm{C} 183 \mu \mathrm{m}$, Hichrom Ltd running at $45^{\circ} \mathrm{C}$.

The ion transition for FF was $\mathrm{m} / \mathrm{z} 539$ to 313 . The validation range of the assay was $10-1000 \mathrm{pg} / \mathrm{mL}$ for FF. Interbatch precision was $\leq$ $16.0 \%$ coefficient of variation $(\mathrm{CV})$ over the assay range; the lower limit of quantification for FF was $10 \mathrm{pg} / \mathrm{mL}$. The conditions for stability for freeze/thaw, matrix, processed extract and long-term stability in frozen matrix were 3 freeze-thaw cycles at $-20^{\circ} \mathrm{C} /$ ambient temperature, 24 hours at ambient temperature, 24 hours at ambient temperature and 412 days at $-20^{\circ} \mathrm{C}$, respectively.

Where reported concentrations were above the higher limit of quantification the plasma samples were diluted, as appropriate, prior to analysis to provide concentrations within the validated range. Quality control results from this study met the acceptance criteria of no more than one third of the quality control results deviating from the nominal concentration by more than $15 \%$, with at least one quality control result acceptable at each concentration. In addition, incurred sample reproducibility was conducted on $6.5 \%$ to $10.2 \%$ of the samples in Study 1 and 2, respectively and $\geq 7.0 \%$ of samples from each of the studies in subjects with asthma. Results showed that $\geq 80 \%$ of the incurred sample results for Studies 1 and 2 and $\geq 66.7 \%$ of the incurred sample results for studies in subjects with asthma were within the limits of $\pm 20 \%$ of the mean of the reanalysis result and its corresponding original result confirming bioanalytical reproducibility in incurred human plasma samples.

\section{Pharmacokinetic analysis}

Pharmacokinetic analyses of plasma FF concentration-time data following inhaled and intravenous administrations to healthy subjects were conducted using non-compartmental Model 200 (for extravascular administration) and Model 202 (for constant infusion), respectively, of WinNonlin Professional Edition Version 5.2 (Pharsight Corporation, Mountain View, CA, USA). Pharmacokinetic variables for all treatments were calculated as follows: $C_{\max }$ and $t_{\max }$ were derived directly from plasma concentration-time profiles, the slowest disposition rate constant $\left(\lambda_{z}\right)$ was calculated by log-linear regression of the terminal portion of the concentration-time profiles where there were sufficient data, $t_{1 / 2}$ was calculated as $0.693 / \lambda,{ }_{z}, U_{(0-t)}$ was calculated using the linear trapezoidal rule for intervals where the concentration data were increasing, and the logarithmic trapezoidal rule for intervals where the concentration data were decreasing, and then extrapolated to infinity using $\lambda_{\mathrm{z}}$ to obtain the $\mathrm{AUC}_{\text {[infinity] }}$. In addition, $\mathrm{AUC}_{(0-12)}$ and $\mathrm{AUC}_{(0-4)}$ were derived in Study 1 for single and repeat dose, respectively and $A \mathrm{UC}_{\left(0-\mathrm{t}^{\prime}\right)}$ was derived for Study 2, where $t$ ' was the common time of the last quantifiable concentration within a subject across all treatments. For intravenous treatment only, total plasma clearance and steady-state volume of distribution (Vss) were determined and mean absorption time (MAT) was derived for inhaled treatment in Study 2.

Population PK modelling of concentration-time data from the asthma study was performed with the computer program NONMEM v7.1.2 (ICON Development Solutions) running in the Predictive Modelling Environment (PME), a UNIX server based environment for NONMEM analysis [referred to as the Pop PK Analysis]. The method selected for minimisation was Stochastic Approximation Expectation Maximization (SAEM) with interaction. Supporting application interfaces for data handling, exploratory diagnostics and simulation included Xpose V4 [18] and R (The R Foundation for Statistical Computing Version 2.10.1 or above). Studies in subjects with asthma (FFA114496 (NCT01431950) [19], HZA106827 (NCT0116513) [20], HZA106829 (NCT01134042) [9], HZA106839 (NCT01018186) [8], HZA106851 (NCT01086410) [14]) were included for the population pharmacokinetic analysis only and no efficacy or safety data for these studies are presented. Given the sparse nature of the sampling in these studies and the high proportion of records reporting FF concentrations below the lower limit of quantification (LLQ; $10 \mathrm{pg} /$ $\mathrm{mL}$ ) particularly at the lower doses, addition of more extensively sampled concentration-time data from a FF/VI study in healthy subjects at a higher dose (800/100) and also 200/25 (HZA102936 [21]) was required to achieve an appropriate structural model to describe the data. FF concentration-time data for the following treatments were included: $100 \mu \mathrm{g} F$ F, $200 \mu \mathrm{g} \mathrm{FF}, 100 / 25 \mu \mathrm{g} F / \mathrm{VI}, 200 / 25 \mu \mathrm{g}$ and $800 / 100 \mu \mathrm{g}$. Configuration (FF single-strip, FF two-strip or FF/VI) was included as a variable. As a consequence of the large extent of nonquantifiable data in each dataset it was necessary to use methodology that maximised the likelihood for all the data, treating those data below the LLQ as censored. The data were analysed using the methodology referred to as $\mathrm{M} 3$ and requires the use of the F_FLAG option and PHI function available in NONMEM v7 [22].

The population pharmacokinetics of FF has previously been reported for the asthma patient data [23] for studies which used the two-strip configuration. The population PK analysis presented here is based on the earlier analysis but also includes study FFA114496 which used the single-strip configuration and allows for evaluation of configuration to be a potential covariate on FF pharmacokinetics. The covariates considered for evaluation of effects on FF pharmacokinetics in the analysis presented here included population (healthy subjects or subjects with asthma), age, weight, height, sex, ethnicity (Hispanic or Latino/non-Hispanic or Latino), race, BMI, PFEV (FEV1 \% predicted), study and configuration (FF single-strip, FF two-strip or FF/VI). Due 
to limited numbers of subjects in some of the race categories subjects were grouped and categorised as 'RACE1' as follows: RACE1 $1=1$ - White Caucasian; RACE1 $1=2$ - East Asian, Japanese and South East Asian; RACE1=3 - African American/African, White Arabic, American Indian/Native Alaskan and Mixed. Plots of inter-subject variability (ETA) versus each covariate were used to select potential covariates for inclusion in the FF PK model. Each potentially significant covariate identified from the plots was individually included on the fixed parameter in the base model to identify significant covariates. Next, all the significant covariates were added to the base model. After the full model had been defined, the significance of each covariate was tested individually by removal one at a time from the full model.

Model evaluation to assess the adequacy of the final model, including the effects of statistically significant covariates was performed using a Visual Predictive Check (VPC) procedure [24]. This procedure was conducted as follows: 1000 replicates of the original dataset were simulated, based on the parameter estimates of the final model, and a $95 \%$ prediction interval computed based on the simulated datasets. The observed plasma concentration-time data was plotted on the prediction interval to visually assess the concordance between the simulated and observed data. In addition the observed proportion of data below the limit of quantification (BLQ) was plotted with the model prediction interval for proportion of the BLQ data to visually assess the concordance between the simulated and observed BLQ data.

\section{Safety evaluations}

Adverse events (AEs), clinical laboratory tests and vital signs were monitored throughout both of the healthy subject studies. In the healthy subject studies a complete physical examination, including 12lead electrocardiogram, was done at screening.

\section{Statistical analysis}

A hypothesis testing approach was used to assess FF dose proportionality. Dose proportionality was to be concluded if the $90 \%$ confidence interval (CI) for the slope from the power model analysis fell within the pre-defined acceptance range $(0.84,1.16)$ for AUC and for $\mathrm{C}_{\max }$, separately. To assess dose proportionality of $\mathrm{FF} \log _{\mathrm{e}}-$

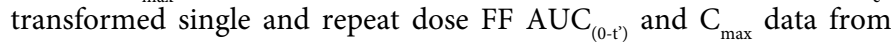
Study 1 were analysed separately using the power model, fitting log (dose) and period as fixed effects, and individual subject intercept as a random effect. An estimate of slope (with corresponding 90\% CI) was calculated.

Analysis of variance (ANOVA) was used as a secondary approach to assess dose proportionality. Following $\log _{\mathrm{e}}$-transformation, single and repeat dose, dose-normalised $\mathrm{AUC}_{\left(0-\mathrm{t}^{\mathrm{r}}\right)}$ and $\mathrm{C}_{\max }$ of $\mathrm{FF}$ were analysed separately using a mixed effects model, fitting dose and period as fixed effects, and subject intercept as a random effect. The reference doses for the ANOVA were FF 600 and $100 \mu \mathrm{g}$ for single and repeat dose, respectively, as these are comprised of the $100 \mu \mathrm{g}$ strength, which is anticipated to be the most commonly, used clinical strength.

For Study 1, $\mathrm{AUC}_{(0-\mathrm{t})}$ from the inhaled and intravenous formulations was used to estimate absolute bioavailability for inhaled FF. The AUC values were dose-normalised, by dividing by nominal dose, then using $\log _{\mathrm{e}}$-transformed values, analysed using a mixed effects model. Mixed models were fitted with treatment as a fixed effect and subject as a random effect. A point estimate and associated $90 \%$ CI was constructed for the difference of inhalation versus intravenous dosing, then exponentially back-transformed to provide corresponding estimates for the ratio.
To assess bioequivalence in Study 2, loge-transformed, $\mathrm{AUC}_{(0-\infty)}$, $\mathrm{AUC}_{\left(0-t^{\prime}\right)}, \mathrm{AUC}_{(0-\mathrm{t})}$ and $\mathrm{C}_{\max }$ of FF, were analysed using a mixed effects model with fixed effect terms for period and treatment. Subject was treated as a random effect in the model. Point estimates and associated $90 \%$ confidence intervals were constructed for the differences "FF single strip - FF two-strip" and "FF single strip - FF/VI", then exponentially back-transformed to provide point estimates and associated $90 \%$ confidence intervals for the treatment ratios. For each comparison of interest, bioequivalence was to be concluded if the $90 \%$ confidence intervals for the ratios of all primary endpoints $\left(\mathrm{AUC}_{(0-\infty)}, \mathrm{AUC}_{\left(0-\mathrm{t}^{\prime}\right)}\right.$, $\mathrm{AUC}_{(0-\mathrm{t})}$ and $\mathrm{C}_{\max }$ ) were completely contained within the range 0.8000 to 1.2500

\section{Results}

\section{Study disposition and demographics}

Thirty-six subjects (30 Caucasian, 4 Asian and 2 mixed race) were enrolled into Study 1 . There were two withdrawals, one subject withdrew prior to period 2 for personal reasons and was lost to follow up and one subject withdrew prior to period 3 due to an adverse event (upper respiratory tract infection). The subjects ( 22 males, 14 females) had a mean [range] age of 45-65 [20] years. Ten subjects took concomitant medications during the study. Seven subjects took paracetamol for headache, one subject took paracetamol for sore throat, one subject took aspirin for headache and one subject took paracetamol, codeine and doxycycline for upper respiratory tract infection. None of these medications are considered to be likely to have affected the study outcome.

Thirty subjects (all Caucasian) were enrolled into Study 2. The subjects ( 15 males, 15 females) had a mean [range] age of 47 64 [25] years. One subject withdrew prior to dosing in period 4 due to a protocol deviation (tested positive for drugs of abuse). Seven subjects took concomitant medications during the study. Six subjects took paracetamol for headache and one subject took multiple medications (heparin fraction sodium salt, ibuprofen, metamozole sodium and pantopraxzole) related to treatment of a fractured humerus. None of these medications are considered to be likely to have affected the study outcome.

\section{Pharmacokinetics}

Fluticasone Furoate Dose Proportionality: The mean plasma concentration versus time profiles for single and repeat dose FF are shown in Figure 1a and 1b, respectively. Whilst plasma concentrations of FF were generally quantifiable in the majority of subjects up to 48 hours following single doses of FF $600 \mu \mathrm{g}$ and $1200 \mu \mathrm{g}$ dosing, and 24 hours following single dose FF $300 \mu \mathrm{g}$, data was more limited following repeat doses of FF 50, 100 and $200 \mu \mathrm{g}$ (Figure 2). On average, the maximum plasma concentrations of $\mathrm{FF}$ were achieved at later times $\left(\mathrm{t}_{\max }\right)$ as the FF dose increased (Table S1).

Following repeat dosing (single inhalation), $\mathrm{AUC}_{(0-24)}$ was estimated for $0 / 35$ subjects receiving $50 \mu \mathrm{g}$ and only $21 / 35$ subjects receiving $100 \mu \mathrm{g}$ due to the number of non-quantifiable concentrations. Therefore, $\mathrm{AUC}_{(0-4)}$ has been used to evaluate dose proportionality for repeat dosing. However, it should be noted, that due to the extent of quantifiable data, $\mathrm{AUC}_{(0-4)}$ was only determined for $21 / 35$ subjects at the $50 \mu \mathrm{g}$ dose and $34 / 35$ subjects at the $100 \mu \mathrm{g}$ dose (Table S1).

Single dose $\mathrm{AUC}_{(0-\infty)}$ was dose proportional, however $90 \%$ confidence intervals for $\mathrm{AUC}_{(0-12)}$ and $\mathrm{C}_{\max }$ slope estimates were not 

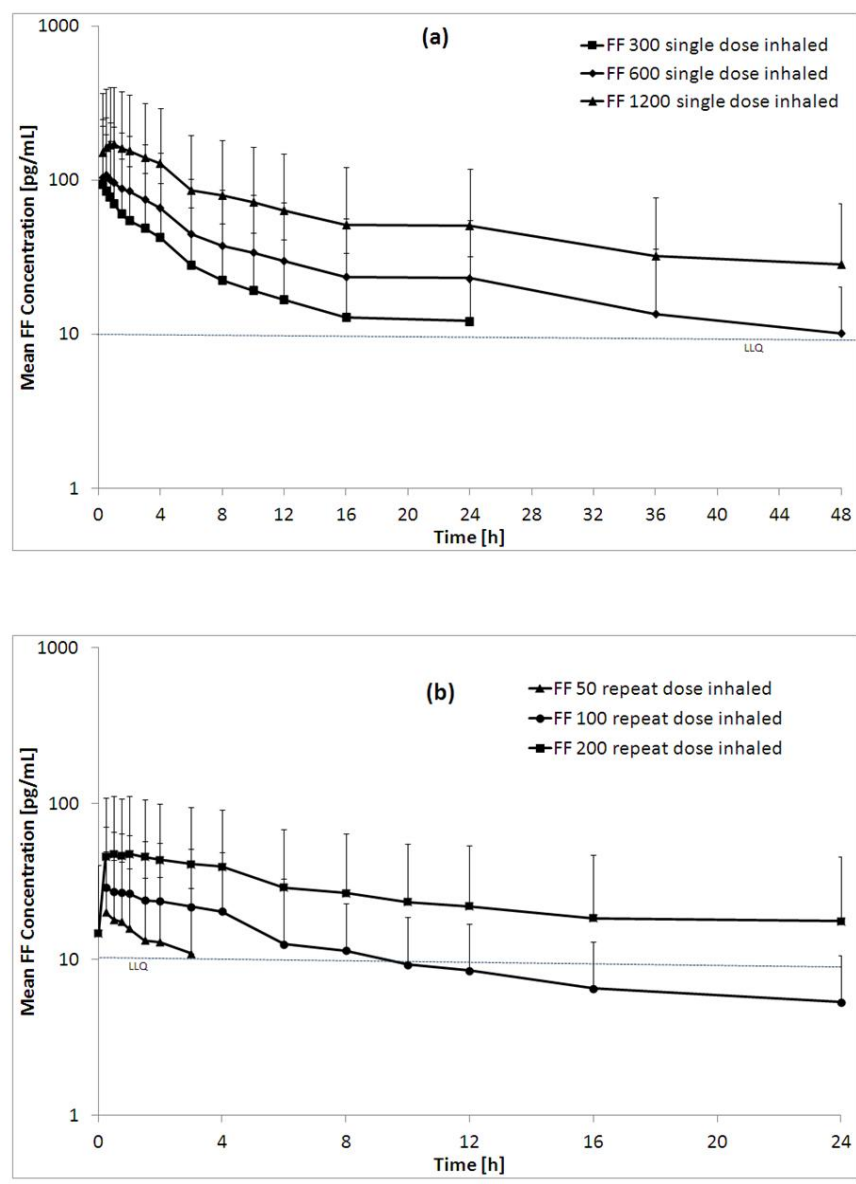

Figure 1: Mean (standard deviation [SD]) fluticasone furoate (FF) semilogarithmic plasma concentration-time profile following single (a) and repeat (b) inhaled administration of FF [Study 1].

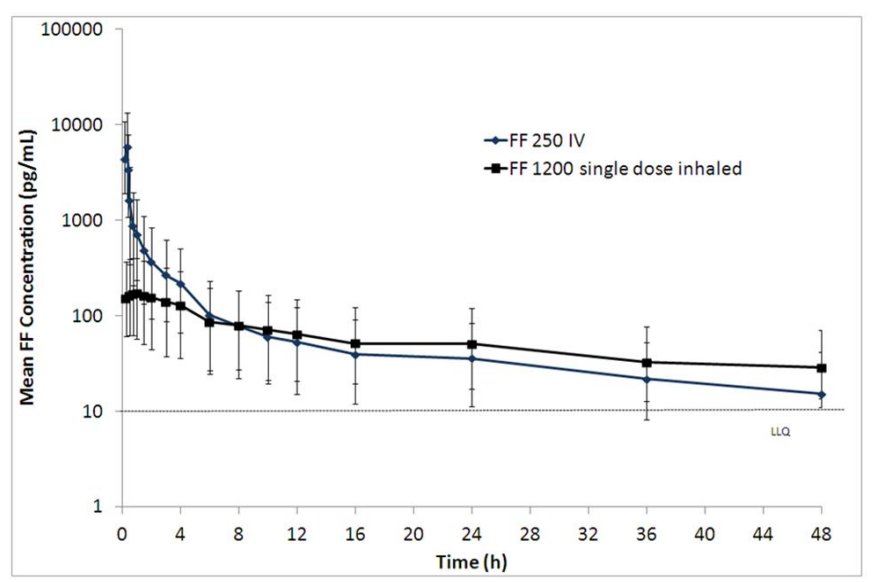

Figure 2: Mean (standard deviation [SD]) fluticasone furoate (FF) semilogarithmic plasma concentration-time profile following single administration of inhaled FF $(1200 \mu \mathrm{g})$ and intravenous FF $(250 \mu \mathrm{g})$ [Study 1].

within the pre-defined acceptance range $(0.84,1.16)$, indicating lack of dose proportionality. Dose proportionality was not demonstrated for repeat dose as the $90 \% \mathrm{CI}$ for the slope for $\mathrm{AUC}_{(0-4)}$ and $\mathrm{C}_{\max }$ did not fall between $(0.84,1.16)$. The estimates of slope suggest less than proportional increases in exposure across the dose range studied, following both single and repeat dosing conditions (Table 1).

Further investigation of dose proportionality using an ANOVA model (data not presented) using FF $600 \mu \mathrm{g}$ single dose and FF 100 $\mu \mathrm{g}$ repeat dose as the reference treatments showed that the lack of dose proportionality for $\mathrm{C}_{\max }$ was across all doses, with a less than proportional increase in $\mathrm{C}_{\max }$ as the dose increased. Consistent with the power model analysis, for single dose $\mathrm{AUC}_{(0-\infty)}$ dose proportionality was demonstrated for each pairwise comparison. The results of the ANOVA for partial AUCs suggest that the lack of dose proportionality seen in the power model is due to the low strength ( $50 \mu \mathrm{g}$ repeat dose and $300 \mu \mathrm{g}$ single dose). The comparisons for AUC between the mid and high strength show little or no deviation from dose proportionality whereas the comparisons between the low and mid strength show less than proportional increase in exposure as the dose increases.

Fluticasone furoate absolute bioavailability: For FF, the estimate of absolute bioavailability of inhaled treatment relative to intravenous treatment was $13.9 \%$. The $90 \%$ CI ranged from $12.7 \%$ to $15.3 \%$ (Table S2).

Fluticasone furoate bioequivalence: Following administration of FF $400 \mu \mathrm{g}$ single strip, plasma concentrations of FF were quantifiable in the majority of subjects up to $24 \mathrm{~h}$ post-dose (29/30 and 25/29 for Dose 1 and Dose 2, respectively) and in approximately half of subjects at the final time point of $36 \mathrm{~h}$ post-dose. Plasma concentrations of FF were also quantifiable in the majority of subjects up to $24 \mathrm{~h}$ postdose following FF $400 \mu \mathrm{g}$ two-strip (22/30 and 23/29 for Dose 1 and 2 , respectively) and FF/VI 400/50 $\mu \mathrm{g},(18 / 30$ and 21/29 for Dose 1 and 2 , respectively). Approximately $1 / 3$ of subjects had quantifiable FF plasma concentrations at $36 \mathrm{~h}$ post-dose following administration of FF $400 \mu \mathrm{g}$ two-strip or FF/VI 400/50 $\mu \mathrm{g}$.

On average the highest FF plasma concentrations, were observed following administration of FF $400 \mu \mathrm{g}$ single strip in both dosing sessions. The lowest FF plasma concentrations, on average, were observed following administration of FF/VI 400/50 $\mu \mathrm{g}$ for both dosing sessions. Plasma FF concentration time profiles and pharmacokinetic parameters were similar for the two dosing occasions of each treatment (Figure 3 and Table S3).

Bioequivalence was not demonstrated for any of the comparisons of interest since the estimated $90 \%$ confidence interval (CI) for the ratio of adjusted geometric means for AUC and $\mathrm{C}_{\max }$ did not fall completely between 0.8000-1.2500 (Table 2).

Population PK analysis: To further investigate the relative bioavailability of the single strip configuration compared with two- strip and FF/VI concentration-time data from clinical studies in subjects with asthma (Figure 4) were subjected to population pharmacokinetic analysis using non-linear mixed effects modelling approach [Pop

\begin{tabular}{|l|c|c|c|c|}
\hline & Parameter & Effect & Point Estimate & $\mathbf{9 0 \%}$ Cl \\
\hline \multirow{3}{*}{ Single Dose } & $\mathrm{AUC}_{(0-\infty)}$ & $\log ($ dose $)$ & 1.040 & $(0.956,1.123)$ \\
\cline { 2 - 5 } & $\mathrm{AUC}_{(0-12)}$ & $\log ($ dose) & 0.775 & $(0.722,0.829)$ \\
\cline { 2 - 5 } & $\mathrm{C}_{\max }$ & $\log ($ dose) & 0.477 & $(0.416,0.539)$ \\
\hline \multirow{2}{*}{ Repeat Dose } & $\mathrm{AUC}_{(0-4)}$ & $\log ($ dose) & 0.758 & $(0.690,0.827)$ \\
\cline { 2 - 5 } & $\mathrm{C}_{\max }$ & $\log ($ dose) & 0.606 & $(0.538,0.674)$ \\
\hline
\end{tabular}

$\mathrm{AUC}=$ area under the curve; $\mathrm{Cl}=$ confidence interval; $\mathrm{C}_{\max }=$ maximum plasma concentration; $\mathrm{FF}$ = fluticasone furoate

Table 1: Results of power model assessment of FF dose proportionality following a single and repeat inhaled administration of FF [dose proportionality Study 1]. 


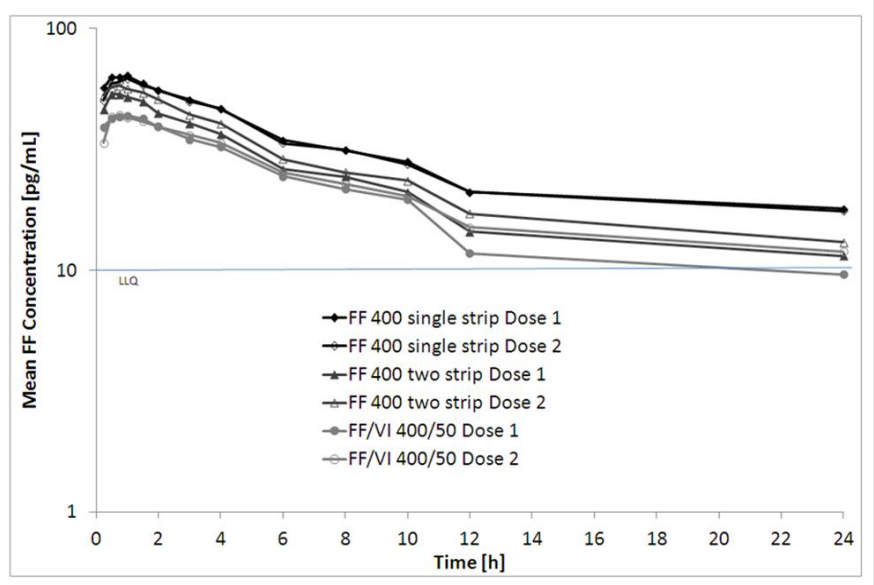

Figure 3: Mean (standard deviation [SD]) fluticasone furoate (FF) semilogarithmic plasma concentration-time profile following single inhaled administration of FF [Study 2].

PK Analysis]. A two compartment linear model, with first order absorption and first order elimination (ADVAN4, TRANS4) was found to describe the FF concentration-time data significantly better than a one-compartment model. Configuration (FF single-strip, FF two-strip or $\mathrm{FF} / \mathrm{VI}$ ) was included as a potential covariate on apparent clearance $(\mathrm{CL} / \mathrm{F})$ and peripheral volume of distribution $(\mathrm{V} 3 / \mathrm{F})$. Although this was not a significant covariate, it was retained in the final model to ensure any small differences were accounted for when generating FF systemic exposure. The goodness of fit plot for the final model appears to provide a reasonable prediction of plasma concentrations of $\mathrm{FF}$ (Figure S1). The plot for the VPC by FF dose and configuration (Figure S2) showed that the majority of the data is captured in the prediction interval that captures $90 \%$ of the population as indicated by the $5^{\text {th }}$ and $95^{\text {th }}$ percentile boundary indicating that the model was valid for this asthma dataset and that the model adequately describes the proportion (\%) of data reported as below the LLQ.

In contrast to the data from the bioequivalence study (Study 2), results of the population $\mathrm{PK}$ analysis show no evidence for a difference in FF systemic exposure following the single-strip configuration, compared with data following the two-strip configuration as $\mathrm{FF}$ or $\mathrm{FF} /$ VI (Table 3). At the FF dose of $200 \mu \mathrm{g}, \mathrm{AUC}_{(0-24)}$ for FF single-strip ELLIPTA (395 pg.h.mL) was, on average, $3 \%$ and $8 \%$ lower compared with FF two-strip ELLIPTA (405 pg.h.mL) and FF/VI (428 pg.h.mL) with considerable overlap in the $95 \%$ CIs. At the lower dose, where data was more censored due to non-quantifiable data, $\mathrm{AUC}_{(0-24)}$ for $\mathrm{FF}$ single-strip ELLIPTA was, on average, $25 \%$ and $23 \%$ lower, compared with FF two-strip and FF/VI.

Safety: Although supra-therapeutic inhaled doses of FF and a high intravenous FF dose were used in these two studies all treatments had a generally good safety profile and there were no new safety issues identified from review of the AE reports. No fatal serious adverse events were reported during the studies. One subject experienced an SAE during the second study which was a 'humerus fracture' which occurred following a fall on slippery ground. The event was not considered related to the study medication and the subject continued in the study. No subjects were withdrawn from either study due to adverse events apart from one subject in Study 1 who was withdrawn due to an adverse event reporting of upper respiratory tract infection that according to the Principal Investigator was drug related. In both studies all AEs were considered by the investigators to be of mild or moderate intensity apart from one report of an $\mathrm{AE}$ of severe intensity in Study 2 (ligament sprain). All AEs, with the exception of the one report of ligament sprain (Study 2) and one report of catheter site pain (Study 1) had resolved by the end of the study.

For Study 1, a total of 159 adverse event (AE) episodes were reported in 33 subjects (92\%) during the study, 8 were reported after administration with FF 300 single dose, 17 were reported after FF 600 single dose, 12 after FF 1200 single dose, 38 after FF 50 repeat dose, 41 after FF 100 repeat dose, 28 after FF 200 repeat dose and 15 after FF 250 IV. The most frequently reported AEs were headache, reported by 14 (39\%) subjects, oropharyngeal pain, reported by $9(25 \%)$ subjects and catheter site reaction, reported by $9(25 \%)$ subjects (Table S4). There was no increase in total or specific AE frequency with increasing FF dose.

Thirteen subjects (36\%) reported 29 AEs that were defined as being of special interest and class related (Table S5). Nine subjects (25\%) reported ororpharyngeal pain, $6(17 \%)$ subjects reported dysphonia and $1(3 \%)$ subject reported rash. All but two of these (rash in one subject [FF 50 repeat dose] and sore throat in one subject [FF 50 repeat dose]) were considered causally related to study drug.

For Study 2, a total of 32 adverse event (AE) episodes were reported in 16 subjects (53\%) during the study, 16 were reported after administration with FF 400 single-strip, 11 were reported after FF 400 two strip and 5 were reported after FF/VI 400/25. The most frequently reported AEs were headache, reported by seven (23\%) subjects in the study, and nasopharyngitis, reported by $4(13 \%)$ subjects (Table S6). Four subjects experienced five AEs that were defined as being of special interest and class related (Table S7). None of these events were considered causally related to study drug. These were; oropharyngeal pain (two subjects [6\%]), rash (one subject [3\%]) throat irritation (one subject [3\%]), and humerus fracture (one subject [3\%]). The report of humerus fracture was a serious adverse event and has been described above.

\section{Discussion}

Dose proportionality was demonstrated for FF following single inhaled dosing since $\mathrm{AUC}_{(0-\infty)}$ showed dose proportionality across the strengths. However, a lack of dose proportionality was seen for the partial AUCs and $\mathrm{C}_{\max }$. $\mathrm{FF}$ is poorly soluble and has been shown previously to exhibit absorption limited pharmacokinetics [25]. Evaluation of dose proportionality using partial AUCs is confounded by this rate-limited absorption. Incomplete absorption, especially using $\mathrm{AUC}_{(0-4)}$ for the repeat dose treatments is reflected in the lack of dose proportionality seen for the partial AUCs. The lack of dose proportionality seen for $\mathrm{C}_{\max }$ has previously been reported following administration of FF/VI [11] and evaluation of dose proportionality using $\mathrm{C}_{\max }$ is also confounded by the rate limited absorption of FF. The less than proportional increases seen in FF systemic exposure are supported by the later $\mathrm{T}_{\max }$ values observed at higher doses, reflecting slower rates of absorption as dose increases. Median $\mathrm{T}_{\max }$ for single dose administration increased from 15 minutes to 30 minutes to 60 minutes as the dose increased. A very similar increase in $\mathrm{T}_{\text {max }}$ was seen for repeat dose administration with median $T_{\max }$ values increasing from 15 minutes to 30 minutes to 45 minutes as the dose increased. FF acts topically in the lung, whilst systemic exposure is related to safety. Consequently, the lack of dose proportionality for $\mathrm{FF} \mathrm{C}_{\text {max }}$ would be considered not to impact efficacy. Furthermore, as the results show a less than dose proportional increase in $\mathrm{FF} \mathrm{C}_{\max }$ this would also be 
Citation: Allen A, Bal J, Moore A, Stone S, Tombs L (2014) Bioequivalence and Dose Proportionality of Inhaled Fluticasone Furoate. J Bioequiv Availab 6: 024-032. doi:10.4172/jbb.1000176

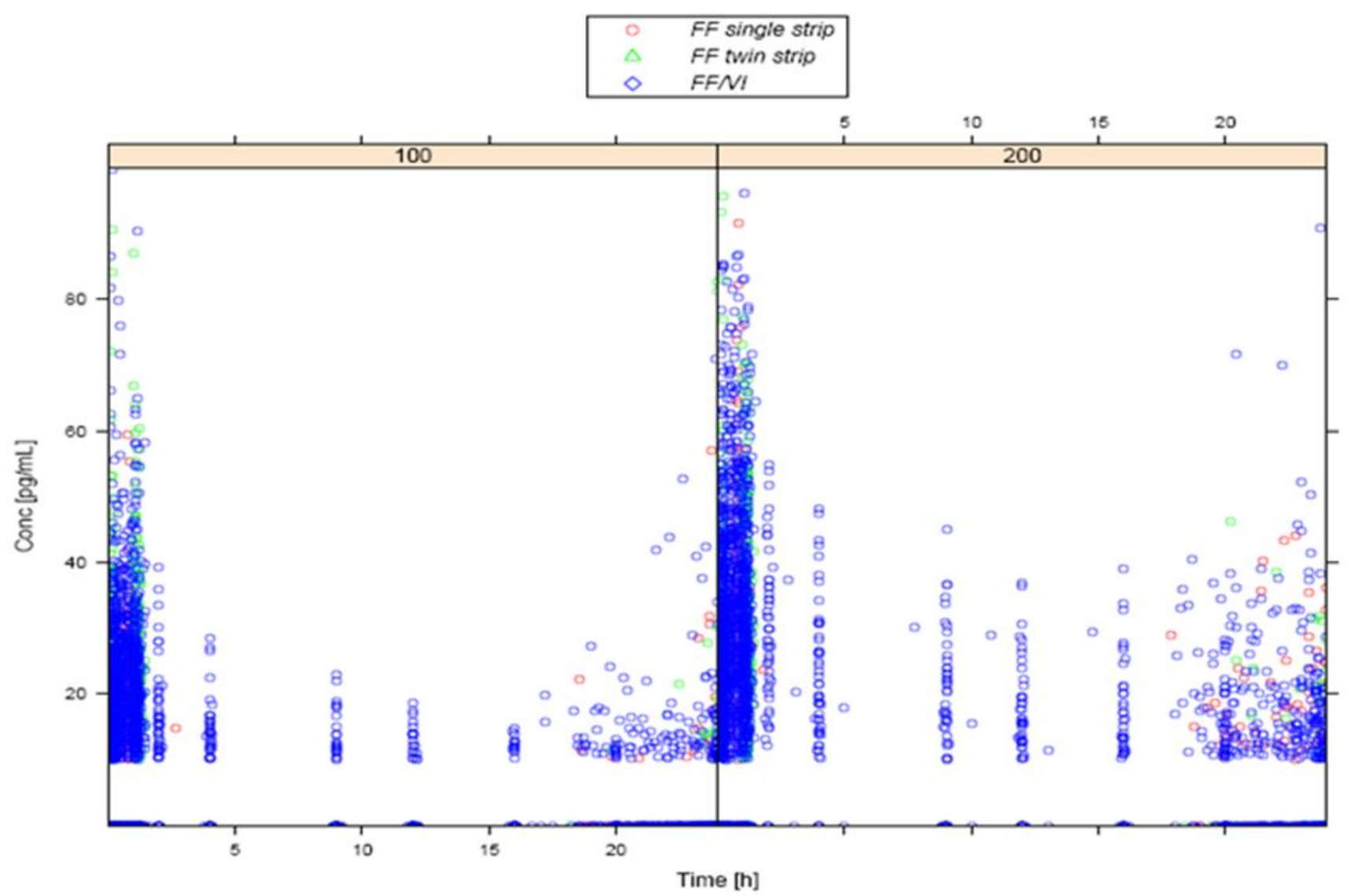

Figure 4: FF concentrations relative to time of last dose following FF (100 and $200 \mu \mathrm{g}$ ) as single and two-strip configuration and FF/VI (100/25 and 200/25) to subjects with asthma [Pop PK Analysis].

\begin{tabular}{|c|c|c|c|c|}
\hline Parameter & Treatment Comparison & Adjusted Geometric Means & Ratio of Adjusted Geometric Means & $90 \% \mathrm{Cl}$ of the Ratio \\
\hline \multirow{2}{*}{$\operatorname{AUC}_{(0-\infty)}$} & FF 400 single-strip / FF 400 two-strip & $1144.7 / 889.5$ & 1.29 & $(1.14,1.46)$ \\
\hline & FF 400 single-strip / FF/VI 400/50 & $1144.7 / 714.8$ & 1.60 & $(1.37,1.87)$ \\
\hline \multirow{2}{*}{$A \cup C_{\left(0-t^{\prime}\right)}$} & FF 400 single-strip / FF 400 two-strip & $560.2 / 458.0$ & 1.22 & $(1.16,1.29)$ \\
\hline & FF 400 single-strip / FF/VI 400/50 & $560.2 / 401.1$ & 1.40 & $(1.31,1.49)$ \\
\hline \multirow{2}{*}{$A \cup C_{(0-t)}$} & FF 400 single-strip / FF 400 two-strip & $723.0 / 531.6$ & 1.36 & $(1.23,1.50)$ \\
\hline & FF 400 single-strip / FF/VI 400/50 & $723.0 / 441.2$ & 1.64 & $(1.44,1.87)$ \\
\hline \multirow{2}{*}{$\mathrm{C}_{\max }$} & FF 400 single-strip / FF 400 two-strip & $67.84 / 60.08$ & 1.13 & $(1.07,1.20)$ \\
\hline & FF 400 single-strip / FF/VI 400/50 & $67.84 / 47.75$ & 1.42 & $(1.33,1.52)$ \\
\hline
\end{tabular}

$A \cup C=$ area under the curve; $\mathrm{Cl}=$ confidence interval; $\mathrm{C}_{\max }=$ maximum plasma concentration; $\mathrm{FF}=$ fluticasone furoate

Table 2: Results of assessment of FF bioequivalence following single inhaled administration of FF [bioequivalence Study 2].

\begin{tabular}{|c|c|c|c|c|c|c|}
\hline \multirow[b]{2}{*}{ Treatment } & \multirow[b]{2}{*}{ Dose $(\mu \mathrm{g})$} & \multirow[b]{2}{*}{$\mathbf{n}$} & \multicolumn{2}{|c|}{$\operatorname{AUC}_{(0-24)}$} & \multicolumn{2}{|c|}{$\mathbf{C}_{\max }$} \\
\hline & & & Geometric Mean [pg.h/mL] & $95 \% \mathrm{Cl}$ [pg.h/mL] & Geometric Mean [pg/mL] & $95 \% \mathrm{Cl}[\mathrm{pg} / \mathrm{mL}]$ \\
\hline FF single-strip & 100 & 116 & 180.7 & $117.4,292.0$ & 27.0 & $15.4,50.3$ \\
\hline FF two-strip & 100 & 186 & 240.9 & $112.5,546.4$ & 32.2 & $19.3,58.0$ \\
\hline $\mathrm{FF} / \mathrm{VI}$ & 100 & 434 & 235.0 & $108.8,505.4$ & 33.8 & $19.8,66.5$ \\
\hline FF single-strip & 200 & 115 & 394.5 & $194.4,917.8$ & 55.1 & $32.6,98.2$ \\
\hline FF two-strip & 200 & 161 & 405.4 & $169.7,990.7$ & 65.9 & $40.3,125.5$ \\
\hline FF/VI & 200 & 432 & 427.5 & $191.7,930.3$ & 68.2 & $39.2,123.7$ \\
\hline
\end{tabular}

Table 3: Summary statistics for FF AUC $\mathrm{C0-24)}$ and $\mathrm{C}_{\max }$ in subjects with asthma by configuration [Pop PK analysis].

considered not to have an adverse impact on safety. Therefore, the lack of dose proportionality for $\mathrm{FF} \mathrm{C}_{\max }$ is considered to be of no clinical relevance.
On average, the absolute bioavailability for FF $200 \mu \mathrm{g}(1200 \mu \mathrm{g}$ dose) was estimated to be $14 \%$ (90\% CI: $13 \%, 15 \%)$ and is similar to that reported for FF (on average 15\%) following inhaled administration 
as FF/VI [11]. Oral bioavailability is approximately $1 \%$ for FF [17] and hence pharmacokinetic data following inhaled dosing represents almost exclusively the dose absorbed from the lung, with negligible contribution to systemic exposure from the swallowed portion of the dose.

Bioequivalence was not demonstrated for FF $400 \mu \mathrm{g}$ (single-strip) compared with either FF $400 \mu \mathrm{g}$ (two-strip) or FF/VI 400/50 $\mu \mathrm{g}$, as the $90 \%$ CIs for the true ratios of the adjusted geometric means for $\mathrm{AUC}$ and $\mathrm{C}_{\max }$ were not completely contained within the range 0.8000 1.25000 , for all primary comparisons of interest. Since AUC ${ }_{(0-\infty)}$ could not be derived for all subjects $\mathrm{AUC}_{\left(0-\mathrm{t}^{\prime}\right)}$ was also derived and analysed to assess bioequivalence. $\mathrm{AUC}_{\left(0-\mathrm{t}^{\prime}\right)}$ is estimated for all profiles in all subjects and the time of the last quantifiable value $\left(t^{\prime}\right)$ was consistent across a subject. Hence $\mathrm{AUC}_{\left(0-\mathrm{t}^{\prime}\right)}$ would be considered the most robust estimate of AUC for analysis of bioequivalence in this study. The majority of missing values were in the FF/VI treatment often due to the lower exposure and hence it is not surprising that there is a bias to a slightly higher point estimate for $\mathrm{AUC}_{(0-\infty)}$. For $\mathrm{AUC}_{(0-\mathrm{t})}$, due to the higher systemic exposure the time of the last quantifiable value will be later for the FF single-strip compared to the other treatments, and again it is not surprising that there is a bias to a slightly higher point estimate for this parameter.

Batches of drug for any inhaled treatment must be within an agreed specification on the range of particles that can reach the lung (i.e., the respirable mass, generally taken as particles of $<4.5$ microns); some batch to batch variability will be seen, but the respirable mass must be within the specified range [26]. The FF single-strip batch used in the bioequivalence study was at the higher end of the range of respirable mass of typical batches and the two-strip configuration (FF or FF/ VI) had an FF respirable mass that was in the mid to low end of the range for typical batches. These differences in the respirable mass of the batches of drug used for the single- and two-strip products used in the bioequivalence study resulted in approximately $20 \%$ more drug being in the respirable fraction for single-strip FF than for two-strip FF or FF/ VI and likely resulted in the lack of bioequivalence seen in the healthy subjects in Study 2.

In the population pharmacokinetic model development configuration (FF single-strip ELLIPTA, FF two-strip ELLIPTA or FF/ $\mathrm{VI})$ was included as a potential covariate on apparent clearance $(\mathrm{CL} / \mathrm{F})$ and peripheral volume of distribution (V3/F) and although this was not a significant covariate, it was retained in the final model to ensure any small differences were accounted for when generating FF systemic exposure. The results of the population PK analysis of concentrationtime data from asthma patients show that there is no evidence for higher systemic exposure with the FF single-strip configuration compared with FF two-strip or FF/VI. Therefore, although the formal bioequivalence study showed higher exposure with FF single-strip ELLIPTA compared with FF two-strip or FF/VI this was attributable to the differences in the respirable mass between the batches used in the bioequivalence study. In contrast the results of the population PK analysis of data in subjects with asthma show that there is no notable difference in FF systemic exposure between the FF configurations (single-strip, two-strip or FF/ $\mathrm{VI}$ ) in the relevant patient population at clinical doses.

Although FF was administered at supratherapeutic doses; there were no apparent dose-related effects and the majority of AEs reported in both studies were classified as mild. There was one SAE reported, however this was not considered related to treatment. The AE profiles seen in the healthy subject studies are similar to those seen previously in healthy subjects where FF was administered alone or in combination with VI [10-13,21].

A potential limitation of these studies was that they were conducted at supra-therapeutic doses of FF in order to produce measurable FF plasma concentrations to provide robust pharmacokinetic data to meet study objectives. However, due to the linear time-independent pharmacokinetic characteristics of $\mathrm{FF}$, results for pharmacokinetic data from higher doses can be interpolated to the clinical doses. Another potential limitation was that Study 1 was conducted in a partrandomised manner (the inhaled treatments were fully randomised however the intravenous treatment was not randomised). The inhaled and intravenous treatment periods had different dosing procedures, sampling requirements, pharmacokinetic sampling time points and treatment administration. Therefore, it was considered more practical to perform the study with all subjects receiving the intravenous treatment in groups to minimise any logistical issues. No significant statistical issues were expected in following this approach.

Study 2 was conducted as a replicate cross-over design such that each treatment was administered on two separate occasions to each subject. The replicate data for each treatment was reproducible; comparable values for Dose 1 and Dose 2 of each treatment were observed for each primary PK parameter, and there was no overlap of values between treatments. This indicates that the lack of bioequivalence between single-strip FF and two-strip FF or FF/VI was due to true treatment differences, and was not attributable to intra-subject variability.

In conclusion, although the formal bioequivalence study showed higher exposure with FF single-strip ELLIPTA compared with FF two-strip or FF/VI, the results of the population PK analysis of data in subjects with asthma show that there is no notable difference in systemic exposure between the FF configurations (single-strip, twostrip or FF/VI). FF systemic exposure, as measured by $\mathrm{AUC}_{(0-\infty)}$, was dose proportional whilst $\mathrm{C}_{\max }$ showed a less than proportional increase and the inhaled absolute bioavailability of FF was $14 \%$.

\section{Acknowledgements}

All studies were funded by GlaxoSmithKline and the sponsor was involved in the design and conduct of the studies, collection of data, and analysis and interpretation of the data. All authors had access to all of the data, were involved in every stage of the preparation of the paper and approved the final version. The sponsor did not place any restriction on authors about the statements made in the final paper. All listed authors meet the criteria for authorship set forth by the International Committee for Medical Journal Editors. L.T., who consults for a company whose services were contracted by the sponsor, performed the statistical analyses for Studies 1 and 2 .

The authors would like to thank: The investigator and staff at the PRA International, Hanzeplein 1 Entrance 23, Groningen, The Netherlands for their help in the conduct of Study 1; The investigator and staff at Parexel International $\mathrm{GmbH}$ Klinikum Westend, Haus 18, Spandauer Damm 130 14050, Berlin, Germany for their help in the conduct of Study 2; York Bioanalytical Solutions Limited, Northminster Business Park, York YO26 6QR, UK for bioanalytical support for the FF and VI analyses for both studies. The subjects and staff who participated in study FFA114496. Editorial support in the form of copyediting was provided by David Cutler, PhD at Gardiner-Caldwell Communications (Macclesfield, UK) and was funded by GlaxoSmithKline. The article processing charge was paid for by GlaxoSmithKline.

\section{References}

1. Biggadike K, Bledsoe RK, Hassell AM, Kirk BE, McLay IM, et al. (2008) $X$-ray crystal structure of the novel enhanced-affinity glucocorticoid agonist fluticasone furoate in the glucocorticoid receptor-ligand binding domain. J Med Chem 51: 3349-3352.

2. Biggadike K (2011) Fluticasone furoate/fluticasone propionate - different drugs with different properties. Clin Respir J 5: 183-184. 
Citation: Allen A, Bal J, Moore A, Stone S, Tombs L (2014) Bioequivalence and Dose Proportionality of Inhaled Fluticasone Furoate. J Bioequiv Availab 6: 024-032. doi:10.4172/jbb.1000176

3. Rossios $\mathrm{C}$, To $\mathrm{Y}$, To $\mathrm{M}$, Ito $\mathrm{M}$, Barnes $\mathrm{PJ}$, et al. (2011) Long-acting fluticasone furoate has a superior pharmacological profile to fluticasone propionate in human respiratory cells. Eur J Pharmacol 670: 244-251.

4. Woodcock A, Bleecker ER, Busse WW, Lotvall J, Snowise NG, et al. (2011) Fluticasone furoate: once-daily evening treatment versus twice-daily treatment in moderate asthma. Respir Res 12: 160.

5. Bleecker ER, Bateman ED, Busse WW, Woodcock A, Frith L, et al. (2012) Once-daily fluticasone furoate is efficacious in patients with symptomatic asthma on low-dose inhaled corticosteroids. Ann Allergy Asthma Immunol 109: 353-358.

6. Busse WW, Bleecker ER, Bateman ED, Lotvall J, Forth R, et al. (2012) Fluticasone furoate demonstrates efficacy in patients with asthma symptomatic on medium doses of inhaled corticosteroid therapy: an 8-week, randomised, placebo-controlled trial. Thorax 67: 35-41.

7. Bateman ED, Bleecker ER, Lotvall J, Woodcock A, Forth R, et al. (2012) Dose effect of once-daily fluticasone furoate in persistent asthma: a randomized trial. Respir Med 106: 642-650.

8. Busse WW, O'Byrne PM, Bleecker ER, Lotvall J, Woodcock A, et al. (2013) Safety and tolerability of the novel inhaled corticosteroid fluticasone furoate in combination with the $\beta 2$ agonist vilanterol administered once daily for 52 weeks in patients >=12 years old with asthma: a randomised trial. Thorax 68: 513-520.

9. O'Byrne PM, Bleecker ER, Bateman ED, Busse WW, Woodcock A, et al. (2013) Once-daily fluticasone furoate alone or combined with vilanterol in persistent asthma. Eur Respir J.

10. Kempsford R, Allen A, Bareille P, Bishop $H$, Hamilton M, et al. (2011) The safety, tolerability, pharmacodynamics and pharmacokinetics of inhaled fluticasone furoate (FF) and vilanterol (VI) are unaffected by administration in combination. Eur Respir J 38: 138.

11. Allen A, Apoux L, Bal J, Bianco J, Moore A, et al. (2013) The pharmacokinetics of fluticasone furoate and vilanterol following single inhaled administration in combination and intravenous administration of individual components in healthy subjects. J Bioequiv Bioavailab 5: 165-173.

12. Kempsford R, Allen A, Bal J, Rubin D, Tombs L (2013) The effect of ketoconazole on the pharmacokinetics and pharmacodynamics of inhaled fluticasone furoate and vilanteroltrifenatate in healthy subjects. Br J Clin Pharmacol 75: 1478-1487.

13. Allen A, Davis A, Hardes K, Tombs L, Kempsford R (2012) Influence of renal and hepatic impairment on the pharmacokinetic and pharmacodynamic properties and tolerability of fluticasone furoate and vilanterol in combination. Clin Ther 34: 2316-2332.
14. Allen A, Schenkenberger I, Trivedi R, Cole J, Hicks W, et al. (2013) Inhaled fluticasone furoate/vilanterol does not affect hypothalamic-pituitary-adrena axis function in adolescent and adult asthma: randomised, double-blind, placebo-controlled study. Clin Respir J 7: 397-406.

15. Lötvall J, Bakke PS, Bjermer L, Steinshamn S, Crim C, et al. (2012) Efficacy and safety of 4 weeks' treatment with combined fluticasone furoate/vilanterol in a single inhaler given once daily in COPD: a placebo-controlled randomised trial. BMJ Open 2: e000370.

16. Boscia JA, Pudi KK, Zvarich MT, Sanford L, Siederer SK, et al. (2012) Effect of once-daily fluticasone furoate/vilanterol on 24-hour pulmonary function in patients with chronic obstructive pulmonary disease: a randomized, three-way, incomplete block, crossover study. Clin Ther 34: 1655-1666.

17. Hughes SC, Shardlow PC, Hollis FJ, Scott RJ, Motivaras DS, et al. (2008) Metabolism and disposition of fluticasone furoate, an enhanced-affinity glucocorticoid, in humans. Drug Metab Dispos 36: 2337-2344.

18. Jonsson EN, Karlsson MO (1999) Xpose--an S-PLUS based population pharmacokinetic/pharmacodynamic model building aid for NONMEM. Comput Methods Programs Biomed 58: 51-64.

19. Woodcock A, Lötvall J, Bleecker ER, Busse WW, O'Byrne PM, et al. (2013) Efficacy and safety of once-daily (OD) fluticasone furoate (FF) in adults and adolescents with moderate-to-severe persistent asthma. Eur Respir J 42: 696s.

20. Bleecker ER, Lötvall J, Bateman ED, Busse WW, Woodcock A, et al. (2012) Efficacy of fluticasone furoate (FF) as a monotherapy and in combination with vilanterol (VI) over 12 weeks in patients with persistent asthma. Eur Respir J 40: P2091.

21. Kempsford R, Allen A, Kelly K, Saggu P, Crim C (2013) A Repeat-dose Thorough QT Study of Inhaled Fluticasone Furoate (FF)/Vilanterol (VI) Combination in Healthy Subjects. Br J ClinPharmacol.

22. Ahn JE, Karlsson MO, Dunne A, Ludden TM (2008) Likelihood based approaches to handling data below the quantification limit using NONMEM VI. J PharmacokinetPharmacodyn 35: 401-421.

23. Allen A, Siederer S, Yang S Population pharmacokinetics of inhaled fluticasone furoate and vilanterol in adult and adolescent subjects with asthma [submitted].

24. Post TM, Freijer JI, Ploeger BA, Danhof M (2008) Extensions to the visual predictive check to facilitate model performance evaluation. J PharmacokinetPharmacodyn 35: 185-202.

25. Allen A, Bareille PJ, Rousell VM (2013) Fluticasone furoate, a novel inhaled corticosteroid, demonstrates prolonged lung absorption kinetics in man compared with inhaled fluticasone propionate. ClinPharmacokinet 52: 37-42.

26. Dolovich M (1999) New propellant-free technologies under investigation. J Aerosol Med 12: S9-17. 